\title{
Prediction of weld shape for fiber laser welding based on hybrid heat source model
}

\author{
Y. Liu ${ }^{1, a}$, P. Jiang ${ }^{1, b^{*}}$, Y.W. Ai ${ }^{1, c}$ and C. Yue ${ }^{1, d}$ \\ ${ }^{1}$ The State Key Laboratory of Digital Manufacturing Equipment and Technology, School of \\ Mechanical Science and Engineering, Huazhong University of Science \& Technology, 430074 \\ Wuhan, PR China
}

ayangliuhust@126.com, bjiangping@hust.edu.cn, caiyuewei1@126.com, dyue30000@126.com

\begin{abstract}
Keywords: Laser welding, Temperature field, Fluent, Weld shape
Abstract. The shape of welded joints directly affects the quality and stability of joints, so the weld shape prediction has great significance and engineering value to for process parameters selection. A mathematical model of fiber laser welding temperature field simulation is proposed for weld shape prediction in this paper. A new hybrid heat source model with double ellipsoid heat source and linear attenuation peak heat is applied, and the simulation process is conducted on Fluent 14.0. The simulation results are in good agreement with the experimental results, which demonstrates the proposed hybrid heat source can more accurately simulate the weld shape. Meanwhile the use of structured hexahedral mesh reduced computer storage space and the amount of computation greatly in ensuring the accuracy, thus raising the simulation speed eventually.
\end{abstract}

\section{Introduction}

Laser welding has been widely applied in the industry as the advantage of high quality, high precision, high performance, high speed, good flexibility, small heat affected zone and low deformation or distortion [1-3].In deep penetration laser welding process, shape of the welded joints directly affects the property and stability of quality of joints [4]. Predicting the weld shape of deep penetration laser accurately under different process conditions will play an important role in ensuring the integrity of the workpiece's weld shape, size, accuracy and reliability of joint quality [5]. Simultaneously it is essential for reducing the time and experimental work to achieve pre-selected process parameters and provide theoretical basis and foundation data.

As the basis of numerical simulation of welding, temperature wield could promptly reflect the complex temperature distribution in welding process and thus reflect weld shape in the heat-affected zone [6]. However, with the property of partial concentrated, transient and fast-moving heat source, it's easy to form large-gradient and uneven temperature field, which will lead to thermal deformation of welded joints during welding process $[7,8]$. Therefore, choosing a reasonable source for the temperature field model to simulate the welding process is a key point for accurate reflection of the heat affected zone and weld shape [9]. Initially two-dimensional Gaussian heat source was introduced, but it was hard to simulate weld shape with large aspect ratio since it didn't consider energy distribution through depth. Later heat source model improved from two-dimension to three-dimension. In 1985, Goldak et al. [10] proposed one double ellipsoid heat source model, the width and depth of the weld bead is close to actual occasion, but the central temperature value is much lower than actual occasion. Sonti et al. [11] proposed exponential attenuating heat model but the predicted weld shape width almost didn't change with the depth. To predict the weld shape more accurately, heat source model improved from one single heat source to hybrid heat source. Amir et al. [9] proposed conical and Gaussian cylindrical hybrid heat source. Although the width and depth is accurate, the simulated weld shape is more like a bowl. Yu et al. [12] studied a hybrid source of double ellipsoid heat source and Gaussian cylindrical heat source, the weld shape is similar to nail head, but the bottom width almost didn't change with depth. Sun et al. [13] reported a hybrid source of Gaussian surface heat source and conical heat source, but the slope of weld changes too sharply and the bottom is unreasonable which didn't match the actual occasion. On the whole, these previous heat source could not simultaneously simulate the similar shape and guarantee the accurate dimensions of weld. 
In this paper, in consideration of the nail head of weld shape, a new hybrid heat source of double ellipsoid heat source and linear attenuation peak heat is proposed to simulate the temperature field for weld shape prediction in the fiber laser welding. Through the comparison with the experimental results, the width and depth of the predicted weld bead as well as the shape is in good agreement with experimental results. The results demonstrates the proposed source model can more accurately predict the weld bead.

\section{Mathematical model}

Due to temperature at designated location keeps fluctuating which causes a relatively complex solution process. Hence, it's essential to conduct a Fluent UDF programming and use a structured hexahedral meshes for solving equations discrete in deep penetration laser welding.

Heat source model. Reasonable selection of laser welding heat source model is able to accurately simulate the welding temperature field and predict weld shape. The most commonly used source models consist of Gaussian surface heat source, rotating Gauss body heat, double ellipsoid heat source, linear attenuation peak body heat, columnar Gaussian heat source and relative hybrid heat source.

Due to a single heat source model cannot simulate the temperature field of laser welding and weld shape well, a new hybrid heat source of double ellipsoid heat source model and linear attenuation peak heat source and observe the effect of adjusting the proportion of two sources on the weld shape. Fig. 1 presents the heat source model in three-dimensional space.

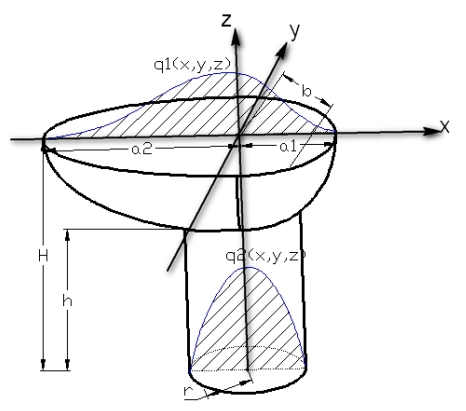

Fig. 1 Hybrid heat source model

The responding energy distribution is shown in Eq. 1.

$$
\left\{\begin{array}{l}
q(x, y, z)=\frac{6 \sqrt{3} f_{1} k_{1} Q}{a_{1} b c \pi \sqrt{\pi}} \exp \left(-3\left(\frac{(x+\mathrm{L}-\mathrm{v} t)^{2}}{a_{1}^{2}}+\frac{y^{2}}{b^{2}}+\frac{z^{2}}{c^{2}}\right)\right) ; \mathrm{H}-\mathrm{h}<\mathrm{z}<\mathrm{H}, \mathrm{x}>0 \\
q(x, y, z)=\frac{6 \sqrt{3} f_{1} k_{2} Q}{a_{2} b c \pi \sqrt{\pi}} \exp \left(-3\left(\frac{(x+\mathrm{L}-\mathrm{v} t)^{2}}{a_{2}^{2}}+\frac{y^{2}}{b^{2}}+\frac{z^{2}}{c^{2}}\right)\right) ; \mathrm{H}-\mathrm{h}<\mathrm{z}<\mathrm{H}, \mathrm{x}<=0 \\
q(x, y, z)=\frac{6 f_{2} Q}{\pi R^{2} H\left(1-e^{-3}\right)} \frac{m z+R}{m H+2 R} \exp \left(\frac{(x+\mathrm{L}-v t)^{2}+y^{2}}{R^{2}}\right) ; r<=R, z<=H
\end{array}\right.
$$

Where $\mathrm{q}(\mathrm{x}, \mathrm{y}, \mathrm{z})$ is the power density at any point inside the source, $Q$ is the effective laser power, $H$ is the total heat loading depth, $h$ is the loading depth of double ellipsoid heat source, $t$ is current time, $v$ is the moving speed of laser, $L$ is the initial location of laser, $f_{1}, f_{2}$ respectively represent proportion of two heat source, and, $f_{1}+f_{2}=1$, while, $k_{1}, k_{2}$ respectively represent proportion of two fractions of double ellipsoid heat source, and $k_{1}+k_{2}=2 . R$ is the radius of linear attenuation peak heat source, $m$ is peak adjustment coefficient. 
Governing equation. In laser welding temperature field simulation, Eq. 2 displays the three-dimensional nonlinear transient heat conduction equations for the problem.

$$
\rho c \frac{\partial T}{\partial t}=\frac{\partial}{\partial x}\left(k \frac{\partial T}{\partial x}\right)+\frac{\partial}{\partial y}\left(k \frac{\partial T}{\partial y}\right)+\frac{\partial}{\partial z}\left(k \frac{\partial T}{\partial z}\right)+Q
$$

Physical model. Based on the experiment and Heat source present in Eqs.1, this paper intend to simulate the temperature of 304 stainless steel laser welding. The work piece's dimension in experiments is $75 \mathrm{~mm} \times 30 \mathrm{~mm} \times 2 \mathrm{~mm}$, laser power is $3 \mathrm{~kW}$, laser's moving speed is $3 \mathrm{~m} / \mathrm{min}$. Since it takes only few milliseconds for the welding to reach quasi-steady state, accounting an appropriate size reduction can guarantee smaller amount of calculation under the premise of sufficient accuracy, the three-dimensional model size established in ICEM CFD is set to $20 \mathrm{~mm} \times 10 \mathrm{~mm} \times 2 \mathrm{~mm}$. To further improve the accuracy and reduce the amount of calculation, structured hexahedral mesh is applied. Mesh is shown as follows (Fig. 2).

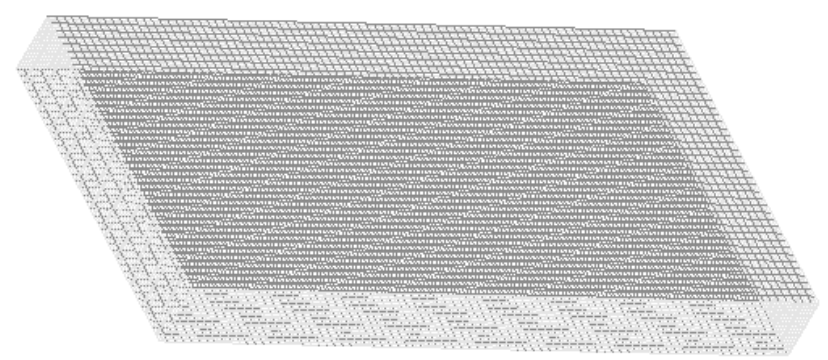

Fig. 2 Structured hexahedral mesh

Boundary conditions. To ensure the divergence of residuals in the iteration process, appropriate boundary conditions is essential to be attached. As heat dissipates mainly through thermal convection and thermal radiation, where heat exchange at high temperature mainly performs as thermal radiation, and otherwise the thermal convection, hence the boundary in three-dimension space is set as follow (Eq. 3):

$$
-k \frac{\partial T}{\partial n}=-q(x, y, z)+h_{c}\left(T-T_{0}\right)+\sigma \varepsilon\left(T_{4}-T_{0}^{4}\right)
$$

Where $k$ is the thermal conductivity, $h_{c}$ is the convection coefficient, $\sigma$ is Boltzmann constant, $\varepsilon$ is thermal radiation coefficient, $T_{0}$ is initial temperature.

This paper intends to set the heat convection coefficient at 30 , the initial temperature of the wall at $300 \mathrm{k}$, and load source term UDF program in the body.

\section{Temperature field simulation results and analysis}

Solution method applies SIMPLE solving algorithm, maximum time step is $0.0005 \mathrm{~s}$, number of time steps is 1000 , and maximum number of iterations per time step is 10 . Corresponding temperature field during the procedure is shown in Fig. 3 


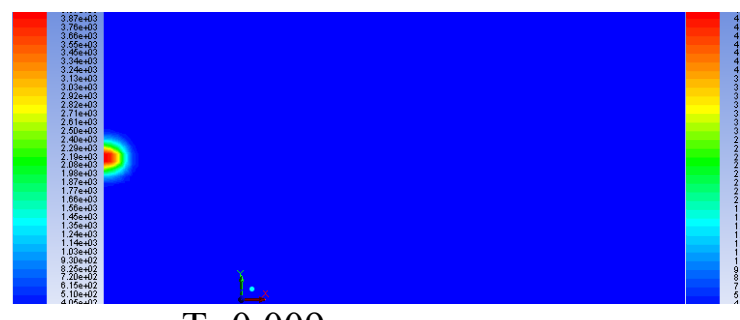

$\mathrm{T}=0.009 \mathrm{~s}$
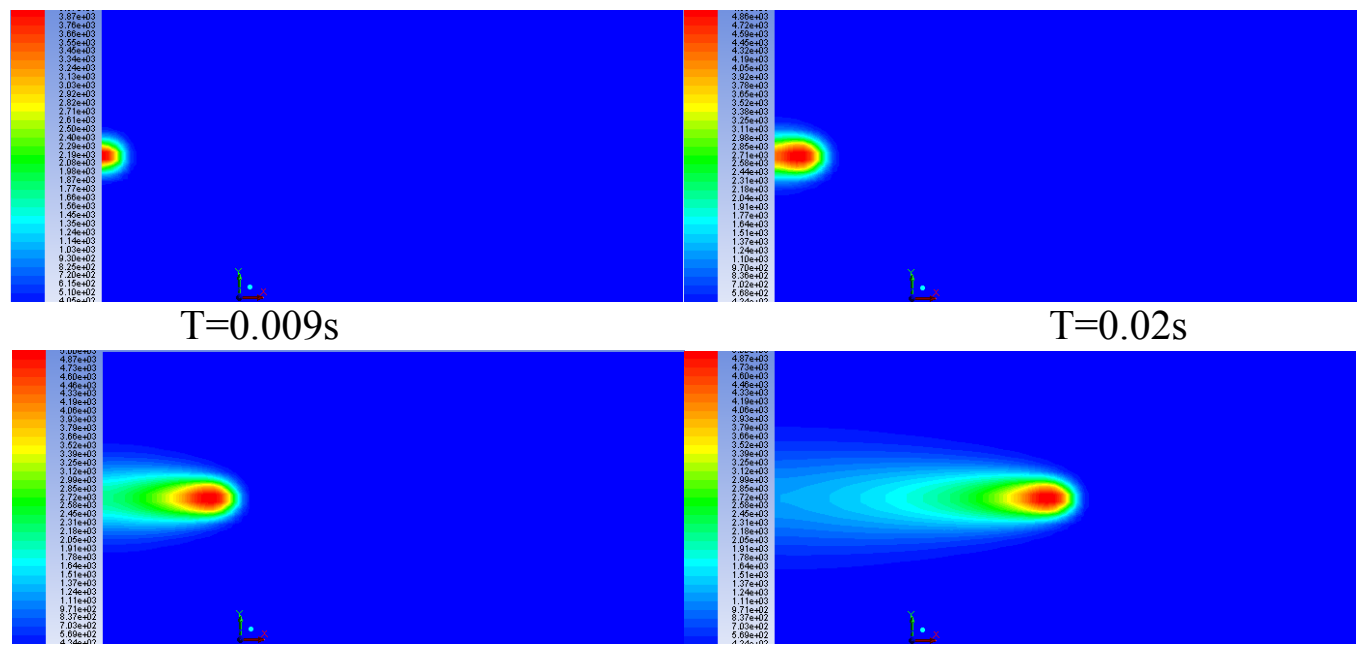

$\mathrm{T}=0.02 \mathrm{~s}$

$\mathrm{T}=0.087 \mathrm{~s}$

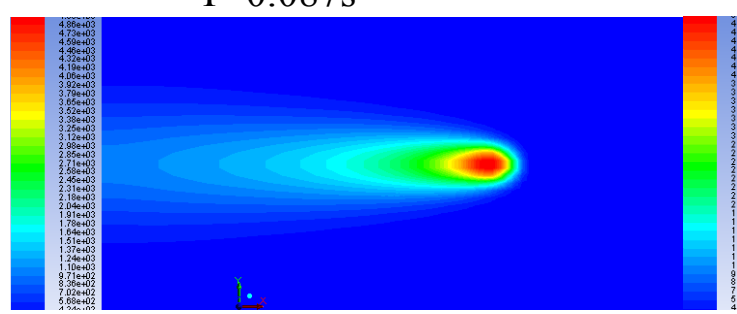

$\mathrm{T}=0.279 \mathrm{~s}$

Fig. 3 Transient temperature field

$\mathrm{T}=0.201 \mathrm{~s}$

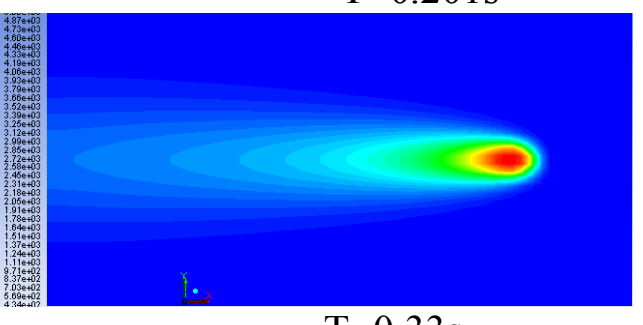

$\mathrm{T}=0.33 \mathrm{~s}$

According to figures above, the temperature field presents an elongated tailing shape, where the front area has a high temperature with intensive isotherm while the back area performs quite the revise, which means the temperature gradient in the front is larger than that at the back, here we can conclude from the phenomenon that as the heat source moving forward, heat transfers much faster at the back on account of continuous heat accumulation.

After using the post-processing software Tecplot to deal with the simulation result file, we got the temperature field of cross section and the simulated weld shape as well as the molten pool, then compared it with the experimental results, as shown in Fig. 4

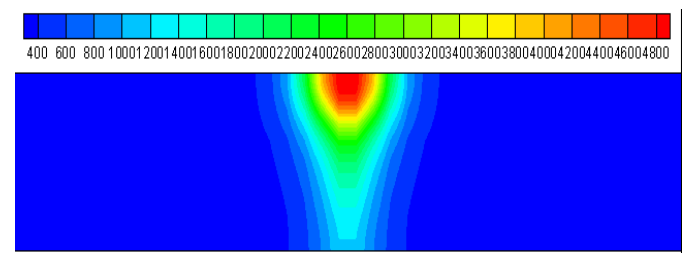

(a)

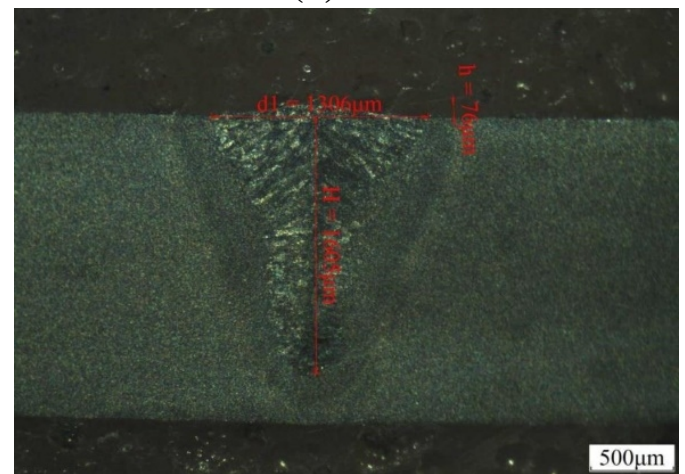

(c)

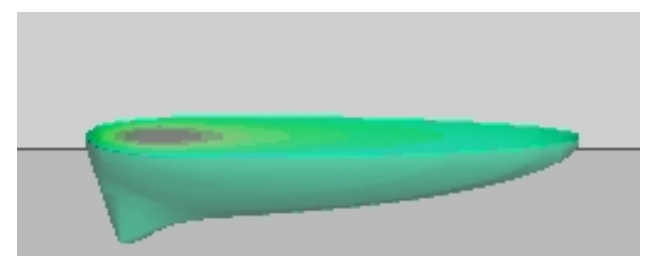

(b)

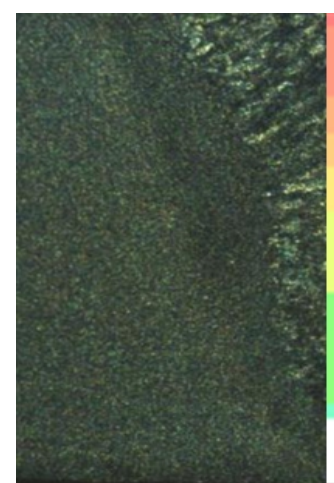

(d)

Fig. 4 The comparison of experimental results and simulation results: (a) Temperature field of cross section; (b) Simulated molten pool; (c) Experimental weld shape; (d) Comparison of practical and simulated weld shape. 
As shown in above figures, the temperature field of cross section represents a nail head shape, where the center of weld shape reaches the highest temperature to $4800 \mathrm{~K}$. As the depth increase, weld width decreases sharply, while in the end weld width seldom decreases. This is precisely consistent with the practical weld bead.

Afterward, the dimensions are measured. The corresponding dimensions of experimental and simulated results, including the errors are listed as follow (Table. 1).

Table. 1 Comparison of dimension and deviation

\begin{tabular}{|c|c|c|c|c|c|c|c|c|}
\hline \multirow[b]{2}{*}{ NO } & \multirow{2}{*}{$\begin{array}{c}\text { Laser } \\
\text { Density } \\
(W)\end{array}$} & \multirow{2}{*}{$\begin{array}{c}\text { Laser } \\
\text { Velocity } \\
(\mathrm{m} / \mathrm{min})\end{array}$} & \multicolumn{3}{|c|}{ Top width(um) } & \multicolumn{3}{|c|}{ Whole Depth(um) } \\
\hline & & & $\begin{array}{c}\text { Experimental } \\
\text { Value (um) }\end{array}$ & $\begin{array}{l}\text { Simulated } \\
\text { Value(um) }\end{array}$ & $\begin{array}{c}\text { Error } \\
(\%)\end{array}$ & $\begin{array}{c}\text { Experimental } \\
\text { Value (um) }\end{array}$ & $\begin{array}{l}\text { Simulated } \\
\text { Value(um) }\end{array}$ & $\begin{array}{c}\text { Error } \\
(\%)\end{array}$ \\
\hline 1 & 2000 & 2.75 & 1306 & 1423 & 8.96 & 1566 & 1652 & 5.49 \\
\hline 2 & 1500 & 3 & 1141 & 1238 & 8.51 & 768 & 827 & 7.69 \\
\hline 3 & 2000 & 2.8 & 1229 & 1333 & 8.49 & 1521 & 1422 & -6.51 \\
\hline
\end{tabular}

From the table, the maximum error between simulated and experimental values is less than $9 \%$, while the minimum error is just $5.5 \%$, here we can get that most of the simulated weld shape dimensions are close to experimental results except the middle width is distinctly larger than experimental result, we guess there are two reasons: for one thing, the proportion and parameters of double ellipsoid heat source is not that reasonably selected, for the other thing, this model neglected the effect of several factors such as surface tension and phase change. However, the simulated weld shape is still quite similar to the experimental results.

\section{Conclusion}

A new hybrid heat source of double ellipsoid heat source and linear attenuation peak heat source is established to simulate the transient temperature for weld bead prediction in fiber laser welding. The dimensions of the simulated weld shape are quite similar with the experimental results of fiber laser welding on the $2 \mathrm{~mm}$ thick stainless steel, the minimum error is simply $5.5 \%$. It's verified that the application of the hybrid heat source of double ellipsoid heat source and linear attenuation peak heat source can accurately simulate practical laser power density and predict the nail head shape of weld. Additionally, corresponding dimensional parameters of weld cross section are quite close to practical results.

\section{Acknowledgements}

This research has been supported by the National Natural Science Foundation of China (NSFC) under Grant No. 51323009, the National Basic Research Program (973 Program) of China under grant No. 2014CB046703, and the Fundamental Research Funds for the Central Universities, HUST: Grant No. 2014TS040. The authors also would like to thank the anonymous referees for their valuable comments.

\section{References}

[1] C. Bagger, F.O. Olsen, Review of laser hybrid welding, Journal of laser applications. 17(2005) $2-14$.

[2] A. Mahrle, E. Beyer, Hybrid laser beam welding-classification, characteristics, and applications, Journal of laser applications. 18(2006) 169-180.

[3] F. Möller, C. Thomy, Interaction effects between laser beam and plasma arc in hybrid welding of aluminum, Physics Procedia. 41(2013) 81-89. 
[4] P. Xia, F. Yan , F. Kong, et al, Prediction of weld shape for fiber laser keyhole welding based on finite element analysis, The International Journal of Advanced Manufacturing Technology. 75(2014) 363-372.

[5] W. Dong, S. Lu, D. Li, et al, Modeling of the weld shape development during the autogenous welding process by coupling welding arc with weld pool, Journal of materials engineering and performance. 19(2010) 942-950.

[6] W.S. Chang, S.J. Na, A study on the prediction of the laser weld shape with varying heat source equations and the thermal distortion of a small structure in micro-joining, Journal of Materials Processing Technology. 120(2002) 208-214.

[7] E. Locke, E.D. Hoag, R. Hella, Deep penetration welding with high-power CO 2 lasers, IEEE Journal of Quantum Electronics. 8(1972) 132-135.

[8] R. Peretz, Workpiece temperature distribution for deep penetration welding with high energy focused beams, Optics and Lasers in Engineering. 7(1987) 69-81.

[9] A.H. Faraji, M. Goodarzi, S.H. Seyedein, et al, Numerical modeling of heat transfer and fluid flow in hybrid laser-TIG welding of aluminum alloy AA6082, The International Journal of Advanced Manufacturing Technology. (2014) 1-16.

[10] J. Goldak, A. Chakravarti, M. Bibby, A new finite element model for welding heat sources, Metallurgical transactions B. 15(1984) 299-305.

[11] N. Sonti, M.F. Amateau, Finite-element modeling of heat flow in deep-penetration laser welds in aluminum alloys, Numerical heat transfer. 16(1989) 351-370.

[12] Y. YU, J. YAN, Y. YIN, et al, Numerical Simulation of the temperature field for Aluminum Alloy Laser-arc Hybrid Welding, Journal of Nanchang Institute of Technology. 4(2013) 56-60.

[13] Q. Sun, M. Li, R. Song, A study on the new heat source in the deep-penetration laser welding. Journal of Welding Technology. 39(2010) 11-14. 